\title{
Inflammatory Cytokines in the Pathogenesis of Pulmonary Arterial Hypertension
}

\author{
Yoshikazu Nakaoka, Tadakatsu Inagaki, \\ and Mikiyasu Shirai
}

\begin{abstract}
Interleukin-6 (IL-6) is a multifunctional pro-inflammatory cytokine elevated in the serum of pulmonary arterial hypertension $(\mathrm{PAH})$ patients and can predict the survival of idiopathic (I)PAH patients. Previous animal experiments and clinical human studies indicate that IL-6 is important in the pathogenesis of PAH; however, the molecular mechanisms of IL-6-mediated pathogenesis of PAH have been elusive. We recently identified IL-21 as a novel downstream cytokine of IL-6-signaling in PAH. First, we found that IL-6 blockade by the monoclonal anti-IL-6 receptor antibody, MR16-1, ameliorated hypoxia-induced pulmonary hypertension (HPH) and prevented the hypoxia-induced accumulation of Th17 cells and M2 macrophages in the lungs. Furthermore, the hypoxia-induced upregulation of IL-17 and IL-21, which are primarily produced by Th17 cells, was also ameliorated by IL-6 blockade in mice. Whereas IL-17 blockade with an anti-IL-17 neutralizing antibody had no effect on HPH, IL-21 receptor-deficient mice were resistant to HPH and exhibited no significant accumulation of M2 macrophages in the lungs. Consistently, IL-21 indeed promoted the polarization of primary alveolar macrophages toward the M2 phenotype. Moreover, significantly enhanced expressions of IL-21 and M2 macrophage markers were detected in the lungs of IPAH patients who underwent lung transplantation. Together, the above data suggest that IL-21 promotes PAH through M2 macrophage polarization, downstream of IL-6-signaling. IL-6/Th17/IL-21-signaling axis might be a novel potential target for treating PAH.
\end{abstract}

Y. Nakaoka $(\bowtie) \cdot$ T. Inagaki

Department of Vascular Physiology, National Cerebral and Cardiovascular Center Research Institute, Suita, Osaka, Japan

e-mail: ynakaoka@ncvc.go.jp

M. Shirai

Department of Advanced Medical Research for Pulmonary Hypertension, National Cerebral and Cardiovascular Center Research Institute, Suita, Osaka, Japan

(C) The Editor(s) (if applicable) and The Author(s) 2020 


\section{Keywords}

Pulmonary arterial hypertension · Inflammation · Cytokine · Interleukin-6 Interleukin-21

\subsection{Background}

Pulmonary arterial hypertension (PAH) is a serious disease characterized by arteriopathy in the small- to medium-sized distal pulmonary arteries, which is associated with arterial muscularization, concentric intimal thickening, and the formation of plexiform lesions [1, 2]. Inflammation and autoimmunity are currently thought of as critical factors to the pathogenesis of PAH [3, 4]. Inflammatory cells such as T cells, $\mathrm{B}$ cells, and macrophages infiltrate the plexiform lesions in patients with advanced $\mathrm{PAH}$. Thus, proinflammatory cytokines produced by these cells may be responsible for the hyperproliferation of pulmonary artery endothelial cells and pulmonary artery smooth muscle cells (PASMCs) [5, 6].

Interleukin (IL)-6 is a multifunctional proinflammatory cytokine linked to numerous autoimmune diseases [7, 8]. Patients with idiopathic (I)PAH exhibit increased IL-6 serum levels, which correlate with their prognoses [3, 9, 10]. Consistent with these findings, lung-specific IL-6 transgenic mice display spontaneous $\mathrm{PH}$ in normoxia and develop greatly exaggerated hypoxia-induced $\mathrm{PH}(\mathrm{HPH})$ [11], whereas IL-6-deficient mice show resistance to HPH [12]. These findings suggest that IL-6 has a significant role in the pathogenesis of PH; however, the downstream target(s) of IL-6 in HPH have been elusive when we started the following study on the pathogenesis of PH. We recently reported that the IL-6/interleukin21(IL-21)-signaling axis played critical roles for the development of HPH in association with M2 macrophage polarization [13]. In this manuscript, we elaborate on the role of IL-6/IL-21-signaing axis in the pathogenesis of PAH.

\subsection{IL-6 in the Pathogenesis of HPH}

We examined the effect of hypoxia (10\% oxygen) on the expression of Il-6 mRNA in the lungs of C57BL/6 mice using quantitative (q)RT-PCR. The $I l-6$ mRNA levels peaked on day 2 and returned to basal levels by day 7 after hypoxia exposure. Next, we investigated the effect of IL- 6 blockade on HPH development by measuring the right ventricular systolic pressure (RVSP), Fulton's Index (right/[left + septum] ventricular weight), and the medial wall thickness index, which is estimated by elastic Van Gieson staining of the pulmonary arterioles. In control antibody-treated mice, hypoxia exposure for 4 weeks induced significant increases in the RVSP, Fulton's Index, and medial wall thickness index compared to the values observed under normoxic conditions. On the other hand, the hypoxia-induced elevation of these parameters was significantly inhibited by treatment with MR16-1. Similarly, thickened medial vascular walls were detected in the lung sections of control 
antibody-treated mice but not in those of MR16-1-treated mice. These data indicate that IL-6 blockade by MR16-1 effectively prevents HPH.

\subsection{IL-21 in the Pathogenesis of HPH}

IL-21 is an IL-2 family cytokine produced by activated T cells, including Th17 cells, that regulates immune responses [14]. We found that the $I l-21 \mathrm{mRNA}$ level peaked on day 2 , remained elevated until day 14 , and returned to the basal levels on day 28 after hypoxia exposure. In addition, hypoxia induced significantly increased numbers of Th17 cells, which produce both IL-17 and IL-21, in the lungs of mice treated with control antibody but not in those treated with MR16-1. These data suggest that IL-6 promotes the accumulation of IL-21-producing Th17 cells in the lungs after hypoxia exposure.

Next, we evaluated the effect of hypoxia on IL-21 receptor (IL-21R) knockout (IL-21RKO) mice [15]. Wild-type (WT) mice exposed to hypoxia for 4 weeks. exhibited significantly increased RVSP, Fulton's Index, and medial wall thickness compared with mice exposed to normoxia. In contrast, the hypoxia-induced elevation of these parameters was significantly inhibited in IL-21RKO mice. Similarly, remodeling of the medial vascular walls following hypoxia exposure in WT mice was also significantly inhibited in the lungs of IL-21RKO mice. These data suggest that IL-21R-deficient mice are resistant to HPH.

Alveolar macrophages have been reported to undergo M2 macrophage polarization in response to hypoxia [16]. We hypothesized that IL-21, which has been reported to be majorly secreted from Th17 cells in mice, might play a role in the hypoxia-induced generation of M2 macrophages in the lung. IL-21 treatment of BALF-isolated alveolar macrophages significantly upregulated the mRNA levels of M2 signature genes, such as Fizz1, Arg1, and Cxcl12, but had no effect on the mRNA levels of M1 signature genes, including Nos $2, I l-12 \beta$, and Tnf- $\alpha$, indicating that IL-21 specifically induces the M2 polarization of alveolar macrophages.

We next examined the effect of IL-21R deletion on the hypoxia-induced upregulation of M2 signature genes, including Fizz1, Arg1, and Cxcl12, in the alveolar macrophages isolated from the BALF after exposure to hypoxia for 4 days. All of these genes were significantly upregulated in the alveolar macrophages isolated from WT mice but not in those from IL-21RKO mice. Similarly, Fizz1 protein expression was increased in the lungs of WT mice but not in those of IL-21RKO mice after exposure to hypoxia for 1 week. In contrast, M1 signature gene expression was comparable in the WT and IL-21RKO mice after exposure to hypoxia. Consistent with these findings, the hypoxia-induced generation of M2 macrophages in the lung was significantly inhibited by treatment with an anti-IL-21-neutralizing antibody but not a control antibody. Collectively, these findings suggest that IL-21 is essential for hypoxia-induced M2 macrophage polarization in the lung.

We next examined the effect of IL-21 blockade on the in vivo hypoxia-induced proliferation of PASMCs. Hypoxia exposure significantly increased the number of anti-Ki67 antibody-positive PASMCs in the lungs of WT mice but not in those of 


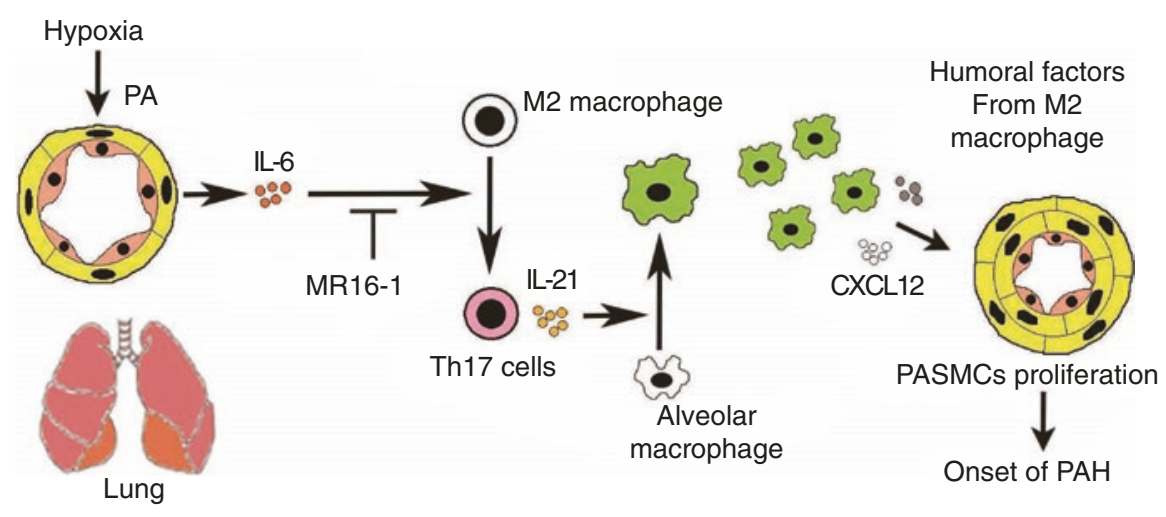

Fig. 20.1 Schematic illustration of the pathogenesis of PAH. IL-6/IL-21-signalling has a central role for the pathogenesis of PAH

IL-21RKO mice. Consistent with this finding, hypoxia exposure significantly increased the number of anti-phospho-Histone $\mathrm{H} 3$ antibody-positive PASMCs in the lungs of mice treated with control antibody but not in those treated with the antiIL-21 neutralizing antibody. Together, these data suggest that IL-21 plays a critical role in the hypoxia-induced proliferation of PASMCs in association with M2 macrophage skewing (Fig. 20.1).

\subsection{Increased Expression of IL-21 and M2 Macrophage Markers in the Lungs of IPAH Patients}

Immunohistochemical analyses of the lung tissues from IPAH patients undergoing lung transplantation showed an excessive infiltration of IL-21-positive cells in the adventitia, obliterative intimal proliferative lesions, and plexiform lesions of the remodeled pulmonary arteries. In contrast, IL-21-positive cell infiltration was barely detected in the lungs of the control patient. We also observed both Arg1-positive and MRC-1-positive cells in all layers of the remodeled pulmonary arteries and alveolar areas examined in the lungs from IPAH patients but not in the tissue from control patients. Taken together, these findings strongly suggest that IL-6/IL-21-signaling axis is essential for the pathogenesis of human PAH in association with M2 macrophage polarization (Fig. 20.1).

\section{References}

1. Pietra GG, Capron F, Stewart S, Leone O, Humbert M, Robbins IM, Reid LM, Tuder RM. Pathologic assessment of vasculopathies in pulmonary hypertension. J Am Coll Cardiol. 2004;43:25S-32S.

2. Rabinovitch M. Molecular pathogenesis of pulmonary arterial hypertension. J Clin Invest. 2012;122:4306-13.

3. Dorfmuller P, Perros F, Balabanian K, Humbert M. Inflammation in pulmonary arterial hypertension. Eur Respir J. 2003;22:358-63. 
4. Schermuly RT, Ghofrani HA, Wilkins MR, Grimminger F. Mechanisms of disease: Pulmonary arterial hypertension. Nat Rev Cardiol. 2011;8:443-55.

5. Tuder RM, Groves B, Badesch DB, Voelkel NF. Exuberant endothelial cell growth and elements of inflammation are present in plexiform lesions of pulmonary hypertension. Am J Pathol. 1994;144:275-85.

6. Tuder RM, Voelkel NF. Pulmonary hypertension and inflammation. J Lab Clin Med. 1998;132:16-24.

7. Kishimoto T. Interleukin-6: Discovery of a pleiotropic cytokine. Arthritis Res Ther. 2006;8(Suppl 2):S2.

8. Kishimoto T, Akira S, Taga T. Interleukin-6 and its receptor: A paradigm for cytokines. Science. 1992;258:593-7.

9. Humbert M, Monti G, Brenot F, Sitbon O, Portier A, Grangeot-Keros L, Duroux P, Galanaud P, Simonneau G, Emilie D. Increased interleukin-1 and interleukin-6 serum concentrations in severe primary pulmonary hypertension. Am J Respir Crit Care Med. 1995;151:1628-31.

10. Soon E, Holmes AM, Treacy CM, Doughty NJ, Southgate L, Machado RD, Trembath RC, Jennings S, Barker L, Nicklin P, Walker C, Budd DC, Pepke-Zaba J, Morrell NW. Elevated levels of inflammatory cytokines predict survival in idiopathic and familial pulmonary arterial hypertension. Circulation. 2010;122:920-7.

11. Steiner MK, Syrkina OL, Kolliputi N, Mark EJ, Hales CA, Waxman AB. Interleukin-6 overexpression induces pulmonary hypertension. Circ Res. 2009;104:236-44, 228p following 244.

12. Savale L, Tu L, Rideau D, Izziki M, Maitre B, Adnot S, Eddahibi S. Impact of interleukin-6 on hypoxia-induced pulmonary hypertension and lung inflammation in mice. Respir Res. 2009;10(6).

13. Hashimoto-Kataoka T, Hosen N, Sonobe T, Arita Y, Yasui T, Masaki T, Minami M, Inagaki T, Miyagawa S, Sawa Y, Murakami M, Kumanogoh A, Yamauchi-Takihara K, Okumura M, Kishimoto T, Komuro I, Shirai M, Sakata Y, Nakaoka Y. Interleukin-6/interleukin-21 signaling axis is critical in the pathogenesis of pulmonary arterial hypertension. Proc Natl Acad Sci U S A. 2015;112:E2677-86.

14. Liu SM, King C. Il-21-producing th cells in immunity and autoimmunity. J Immunol. 2013;191:3501-6.

15. Ozaki K, Spolski R, Feng CG, Qi CF, Cheng J, Sher A, Morse HC 3rd, Liu C, Schwartzberg PL, Leonard WJ. A critical role for il-21 in regulating immunoglobulin production. Science. 2002;298:1630-4.

16. Vergadi E, Chang MS, Lee C, Liang OD, Liu X, Fernandez-Gonzalez A, Mitsialis SA, Kourembanas S. Early macrophage recruitment and alternative activation are critical for the later development of hypoxia-induced pulmonary hypertension. Circulation. 2011;123:1986-95.

Open Access This chapter is licensed under the terms of the Creative Commons Attribution 4.0 International License (http://creativecommons.org/licenses/by/4.0/), which permits use, sharing, adaptation, distribution and reproduction in any medium or format, as long as you give appropriate credit to the original author(s) and the source, provide a link to the Creative Commons license and indicate if changes were made.

The images or other third party material in this chapter are included in the chapter's Creative Commons license, unless indicated otherwise in a credit line to the material. If material is not included in the chapter's Creative Commons license and your intended use is not permitted by statutory regulation or exceeds the permitted use, you will need to obtain permission directly from the copyright holder.

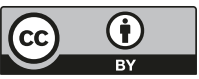

